\title{
INDUSTRIA DE BIOINSUMOS DE USO AGRÍCOLA EN COLOMBIA
}

\section{BIOINOCULANTS INDUSTRY FOR AGRICULTURAL USE IN COLOMBIA}

\author{
Diana Corina Zambrano-Moreno¹, Luisa Fernanda Ramón-Rodríguez ${ }^{2}$, Mario Van Strahlen-Pérez ${ }^{3}$, \\ Ruth Rebeca Bonilla-Buitrago ${ }^{4}$
}

\begin{abstract}
${ }^{1}$ Microbióloga Industrial, M.Sc., cPh.D. en Ciencia Naturales para el Desarrollo -Instituto Tecnológico de Costa Rica. corina_zambrano@yahoo.es; ${ }^{2}$ Microbióloga Industrial, Estudiante de Maestría en Ciencias Biológicas. Iramon@javeriana.edu.co; ${ }^{3}$ Biólogo, M.Sc., Coordinador área de registro y Control de Bioinsumos de uso Agrícola, Instituto Colombiano Agropecuario - ICA. mario.vanstrahlen@gmail.com ${ }^{4}$ Licenciada en Biología y Química, M.Sc., Ph.D., Directora Laboratorio de Suelos, Corporación Colombiana de Investigación Agropecuaria - CORPOICA. rbonilla@corpoica.org.co
\end{abstract}

Rev. U.D.C.A Act. \& div. Cient. 18(1): 59-67, Enero-Junio, 2015

\section{RESUMEN}

Los bioinoculantes son producidos principalmente a partir de bacterias promotoras de crecimiento vegetal. Estos microorganismos agrupan diferentes géneros, con capacidad de estimular e incrementar el crecimiento, la productividad vegetal y controlar otros organismos. El uso de este tipo de microorganismos, en la agricultura colombiana, tiene potencial para generar transformaciones importantes en los sistemas productivos. En este trabajo, la industria de bioinsumos en Colombia fue caracterizada a partir de tres parámetros i. el análisis de los registros ante el Instituto Colombiano Agropecuario - ICA, ii. las patentes solicitadas ante la Superintendencia de Industria y Comercio - SIC y, iii. la solicitud de acceso a recurso genético, desde el 2004 hasta el año 2011 ante el Ministerio de Ambiente y desarrollo sostenible -MADS. En Colombia, se encontraron 111 registros de bioinsumos, destinados, principalmente, a cultivos ornamentales $24,7 \%$, hortalizas $16,3 \%$ y cereales $13,2 \%$. A nivel de patentes, se identificaron 13, de las cuales, 9 se encuentran dentro de la Clasificación Internacional de Patentes - IPC - reconocidas por la Organización para la Cooperación y el Desarrollo Económicos -OCDE- . La base de datos de acceso a recurso genético del MADS permitió identificar 16 solicitudes. Sólo un microorganismo (Bacillus thuringiensis) tuvo registro en las tres instituciones. El estudio permitió concluir que el desarrollo de bioinsumos en Colombia es incipiente, en el ejercicio de estimular investigación para el uso de la biodiversidad microbiana, así como los procesos de registro de patentes, alrededor de los procesos de producción.
Palabras clave: Bio-inoculantes, bacterias promotoras de crecimiento vegetal (PGPR), agricultura ecológica, desarrollo sostenible.

\section{SUMMARY}

Bio-products are primarily produced by using plant growthpromoting bacteria. This group of microorganisms is composed of different genera with the capability to stimulate plant growth increasing their productivity and promoting bio-control. The use of such microorganisms in Colombian agriculture has been highlighted to generate significant changes in production systems. The industry of bio-products was characterized using three parameters: i. records of bioproducts at the Colombian Agricultural Institute -ICA, ii. the patents recorded at the Superintendence of Industry and Commerce -SIC and iii. the Accession request to the genetic resources tool at the Ministry of Environment, Housing and Territorial Development - MADS, within 2004-2011. One hundred eleven records of bio inoculants were found in Colombia, destined mainly to ornamental crops (24.7\%), vegetables (16.3\%), and cereals (13.2\%). At patent level thirteen ones were identified, nine found in the International Patent Classification IPC - recognized by the Organization for Economic Cooperation and Development - OECD. The access to the database of MADS genetic resource allowed to identify 16 applications. Only one microorganism Bacillus thuringiensis was registered at all the assessed institutions. It was concluded that the development of bio-products in Colombia is only emerging in the encouragement research of the use of the microbial biodiversity, as well as the processes regarding patent register of production processes. 
Key words: Bio-inoculants, plant growth promoting bacteria (PGPB), organic farming, sustainable development.

\section{INTRODUCCIÓN}

El desarrollo de los bioinsumos implica el estudio de la diversidad microbiana asociada a cada tipo de suelo y a los cultivos vegetales específicos, así como a los procesos ecológicos inherentes. Este desarrollo puede tomar entre cinco y diez años y su calidad debe ser garantizada, ya que solo así se obtienen los buenos resultados esperados por los agricultores. Hodson \& Díaz (2013) plantean que es necesario cumplir con los requisitos básicos de calidad ante las entidades regulatorias de cada país, para evitar la pérdida de confianza en los productos biológicos.

Las bases del diseño jurídico e institucional, en materia de acceso a recurso genético, incluidos los microorganismos, se encuentran en el Convenio de Diversidad Biológica -CDB-. Posteriormente, la Cumbre Mundial sobre el Desarrollo Sostenible, celebrada en Johannesburgo, en 2002, hizo un llamado para negociar, dentro del marco del CDB, un régimen internacional, que promueva y salvaguarde la participación justa y equitativa en los beneficios derivados de la utilización de los recursos genéticos (Secretaría del CDB, 2011).

En el 2010 se adoptó el Protocolo de Nagoya, que es un acuerdo internacional que tiene por objetivo garantizar una participación justa y equitativa de los beneficios derivados de la utilización de los recursos genéticos, mediante, entre otras cosas, un acceso adecuado a estos recursos y una transferencia apropiada de las tecnologías relacionadas (Secretaría del CDB, 2011).

En 1996 la Comunidad Andina fue el primer bloque de integración regional, que aprobó legislación con respecto al acceso a los recursos genéticos y el reparto equitativo de beneficios, a través de la Decisión 391 de la Comunidad Andina de Naciones - CAN (Ruíz, 2010). En el caso colombiano, la adopción de un régimen de acceso por vía de la Decisión 391 y la falta de reglamentación nacional, se han convertido en una limitación para la investigación y el desarrollo científico Nemogá (2009). Las dificultades para la operación del sistema de acceso tienen como consecuencia, que parte sustancial de la investigación sobre la diversidad biológica y genética del país, se desarrolla por fuera del marco jurídico (Nemogá, 2010).

La Decisión 486 de la CAN estableció un régimen común de propiedad intelectual, en la cual, se prohíbe, expresamente, patentar plantas, animales y procedimientos que sean biológicos o microbiológicos. En 2008 fue emitida la Decisión 689, que modifica la Decisión 486, luego de un proceso solicitado por Perú, para poder profundizar y desarrollar la pro- tección en materia de propiedad industrial y cumplir con sus compromisos adquiridos dentro del acuerdo de promoción comercial, suscrito con EE.UU (Toro, 2010).

El decreto 309 del 2000, la resolución 68 de 2002 y el decreto 302 de 2003 del Ministerio de Ambiente y Desarrollo Sostenible -MADS establecen, en Colombia, el procedimiento de presentación de la solicitud, trámite y obtención del permiso de estudio, con fines de investigación científica, en diversidad biológica. El decreto 1376 del 2013 reglamentó la obtención del permiso de recolección de especímenes silvestres de la diversidad biológica, con fines de investigación científica más no comercial, antes contenida en el permiso de estudio, con fines de investigación científica, regulado por el decreto 309 de 2000; sin embargo, las disposiciones de este decreto no aplican a la recolección de especímenes de especies silvestres de la diversidad biológica, con fines industriales, comerciales o de prospección biológica.

El Instituto Colombiano Agropecuario-ICA es el responsable de adoptar las medidas necesarias en Colombia, para el efectivo control de la sanidad animal y vegetal y de ejercer el control técnico de los insumos agrícolas; la resolución 698 de 2011 establece los requisitos para el registro y control de bioinsumos, incluyendo los microorganismos modificados genéticamente, los cuales habían sido excluidos específicamente en la normatividad anterior.

Según el Conpes 3577 (2009), en los actuales sistemas de producción agrícola, los insumos representan el mayor costo para los agricultores, afectando la producción, puesto que sus precios además, de ser elevados, no son constantes. Una de las posibilidades es explorar las reservas microbiológicas nativas, debido a que ofrecen un potencial para desarrollar tecnologías alternativas para los agricultores, quienes demandan tecnologías accesibles, que no representen un riesgo para el ecosistema o la salud.

Hodson \& Díaz (2013) reconocen avances puntuales en el desarrollo de bioinsumos en Latinoamérica, que podría llevar a esta región a ser un referente mundial en agricultura sostenible, basada en el uso de estos productos biológicos. Dos ejemplos que ilustran la productividad y la competitividad de las industrias agrícolas, contemplan la soya en Suramérica, que, en su mayoría es fertilizada con inoculantes de alta calidad, producidos a partir de Bradyrhizobium japonicum (Shiri-Janagard et al. 2012; Penna et al. 2011) y en México, gran parte de los cultivos de maíz son biofertilizados con la bacteria fijadora de nitrógeno Azospirillum brasilense (García-Olivares et al. 2012).

En Colombia, el uso de bio-fertilizantes ha venido en ascenso, en especial, en el sector arrocero (Arévalo, 2009). Este aumento en el uso de insumos biológicos, se debe, en parte, 
a la existencia de productos en el mercado; por ejemplo, los productos elaborados por la empresa Biocultivos S.A., una empresa de base tecnológica que surgió de los convenios de investigación y desarrollo, establecidos con el Instituto de Biotecnología de la Universidad Nacional de Colombia -IBUN- , el sector privado y el gremio arrocero. Dentro de este proceso, se logró el desarrollo de los productos Fosfosol SW (Penicillium janthine-llum), Fosfosol SC (Penicillium janthinellum), Trifesol WP (Trichoderma viride) y Trifesol SC (Montoya, 2010).

El objetivo central de este trabajo fue caracterizar la industria de bioinsumos en Colombia, a partir de la revisión de los bioinsumos registrados ante el ICA, las patentes solicitadas ante la Superintendencia de Industria y Comercio y la solicitud de acceso a recurso genético ante el MADS, desde el 2004 hasta el año 2011. La comparación de estos tres factores permitió determinar si estos productos biotecnológicos han cumplido con los procesos necesarios para el desarrollo y comercialización, desde la legislación nacional hasta la innovación, a través del uso de la biodiversidad.

\section{MATERIALES Y MÉTODOS}

Caracterización de las empresas productoras de bioinsumos y los bioinsumos registrados ante el ICA; se realizó a partir de la información archivada en las instalaciones de la Dirección General del ICA, este acceso fue autorizado por la Subgerencia de Protección Vegetal, bajo la salvedad que la información del proceso de formulación de las diferentes presentaciones era de extrema confidencialidad y el titular del registro gozaba el derecho de manejarlo como secreto industrial.

La información fue obtenida en Microsoft ${ }^{\circledR}$ Excel 2010, registrando los siguientes datos de las empresas: tipo de actividad desempeñada como productor o importador, tipo de bioinsumo, lugar de registro de la operación de la planta y tipo de empresa. Además, se realizó la caracterización de los productos registrados, tomando la información: Tipo de bioinsumo, microorganismo utilizado y el sistema de producción agrícola de aplicación. La base, se usó para obtener porcentajes estadísticos y observar tendencias en cada factor.

Análisis de patentes: La información fue obtenida a partir de la base de datos de la Superintendencia de Industria y Comercio -SIC- de Colombia. Se utilizaron, como criterios de búsqueda, las palabras microorganismo, bioinsumo e inoculante, contenidas en el título de la patente, teniendo en cuenta las patentes abandonadas, en trámite y concedidas. Luego, se realizó una revisión de las patentes obtenidas, haciendo un filtro, seleccionando las que correspondan a bioinsumos de uso agrícola.
El tratamiento y análisis de la información, se efectuó utilizando el software PAJEK versión 6 XXL, para el comportamiento de las relaciones que se utilizó como indicadores para el análisis de la información, de acuerdo a los siguientes grupos:

a) Análisis básico: indicadores relacionados directamente con las frecuencias involucradas, para los casos de año de otorgamiento, área de conocimiento y el código IPC.

b) Análisis detallado: define los indicadores, que marcan las tendencias y agrupamientos estratégicos; para los casos de la cartera de patentes de las organizaciones/inventores, distribución de las temáticas, según la IPC; los agrupamientos, según el tipo de patente y el IPC. Esta técnica, se aplicó bajo el principio de que la producción científica documental es un indicador de la base científica y la producción de documentos de patentes es un indicador de la capacidad de desarrollo y cambio tecnológico (Díaz et al. 2007).

Delimitación de las patentes en biotecnología. Se utilizó la definición de patente biotecnológica OCDE, que comprende un conjunto de 30 códigos IPC (por sus siglas en inglés International Patent Classification).

Análisis de los trámites de acceso a recurso genético para la investigación o desarrollo: A través del sitio web del MADS, se identificaron los registros que se encontraron sobre solicitudes que involucraran la investigación y desarrollo tecnológico acerca de microorganismos. Se analizaron las solicitudes de acceso presentadas durante este período de tiempo para microorganismos, utilizando el software Vantage Point 32-bit versión 7.1. La información obtenida entre los microorganismos fue cotejada, para verificar el origen del material utilizado para el desarrollo de los productos.

\section{RESULTADOS Y DISCUSIÓN}

Caracterización de las empresas productoras de bioinsumos y los bioinsumos registrados ante el ICA

En Colombia desde el 2004 hasta el 2011, se encontraron 111 registros de empresas de bioinsumos. Este reporte es superior a lo reportado por Nakkeeran et al. (2006), en Estados Unidos, con 33 productos de bacterias promotoras de crecimiento vegetal, registrados para su uso comercial en invernadero y campo. Del total empresas evaluadas en Colombia, el $64,5 \%$ son productoras y un $35,5 \%$, son importadoras. Se observó que el porcentaje de empresas importadoras es alto, resultados que coinciden con el planteamiento de Nemogá (2010), quien hace un llamado a que los investigadores nacionales se pregunten el potencial industrial de los resultados de sus investigaciones, dentro del marco legal o institucional, de lo contario, los productos de investigaciones foráneas son las que hallarán potenciales aplicaciones y obtendrán todos los beneficios. 
Fueron encontrados registros de empresas productoras en 12 departamentos colombianos. La mayoría de estas empresas y de los lugares de operación de las plantas, se encuentran ubicadas en los departamentos de Cundinamarca, Valle del Cauca y Antioquia, con una distribución, en cuanto a empresas, del $45,9 \%, 19,8 \%$ y $12,6 \%$, respectivamente. Esto puede ser explicado, según Cortés \& Vargas (2012), porque estos tres departamentos concentra la mayor población y pertenecen a los cuatro mercados principales del país; además, sugieren que las diferencias institucionales de largo plazo dilucidan las desigualdades regionales actuales.

Las empresas, se encuentran distribuidas en sector privado, con una participación del $94,6 \%$ y, el sector público y mixto, con un 2,7\%, cada uno. Nemogá et al. (2014) registraron que durante las fases de aislamiento de caracterización y de cultivo de microorganismos, participan, equitativamente, laboratorios financiados con recursos públicos o privados. Asimismo, Cabrera \& López (2008) resaltaron la dificultad de separar la investigación básica de la comercial, debido a que la inversión de capital privado, cambia la dinámica y los estándares de difusión de los resultados científicos, porque es cada vez más generalizada la confidencialidad y las restricciones derivadas sobre propiedad intelectual. Por lo tanto, el éxito y la comercialización de las cepas depende de los vínculos entre las organizaciones científicas y las industrias (Bhattacharyya \& Jha, 2012).

Tipos de bioinsumos: De acuerdo a la clasificación del ICA, se ubicaron registros del $46,7 \%$, para las empresas que ofrecen agentes biológicos para el control de plagas y, del $26,7 \%$, de inoculantes biológicos y una baja participación de los extractos vegetales, 19,1\% y productos bioquímicos, con el 7,5\%. Este comportamiento de un mayor número de agentes biológicos coincide con lo reportado por Leng et al. (2011), quien menciona que en la etapa inicial, pocos biopesticidas se registraron en los países desarrollados y, luego, esto incremento, considerablemente; por ejemplo, en Estados Unidos, sólo se registraron 16, en 1996, mientras que 1.090 productos habían sido registrados a finales de 2003. Igualmente, sucedió en China: en el 2008 había 327 bioplaguicidas, que representan el $1,6 \%$ del total de pesticidas registrados. En China, los nuevos desarrollados y bioplaguicidas registrados están aumentando a un ritmo del $4 \%$ cada año y la cuota de mercado de bioplaguicidas subirá el $30 \%$ (Cheng et al. 2010).

En Colombia, se han desarrollado diversos programas para el manejo integrado de plagas, donde los hongos entomopatógenos, juegan un papel muy importante, como controladores biológicos. Inicialmente, la comercialización de productos, con base en hongos entomopatógenos, se vio limitada, por la calidad (Góngora et al. 2009). Para corregir esto y poder garantizarle al agricultor la oferta de un buen producto, el ICA implementó normas de control de calidad. Góngora et al. (2009) reportan que, en el caso de los caficultores que utilizan Beauveria bassiana para el control de la broca del café Hypothenemus hampei, además de exigir el registro ante el ICA, tienen presente la fecha de vencimiento del producto.

Microorganismos empleados: Dentro de los microorganismos más empleados, se encuentran cinco grupos morfológicos: hongos filamentosos, hongos micorrízicos, bacilos Gram positivos, bacilos Gram negativos y levaduras (Cuadro 1). Predominan los hongos filamentosos, con un $59,6 \%$, seguido por los hongos micorrízicos, con un 18,6\%. Según Kadian et al. (2013), los hongos micorrízicos y hongos como Trichoderma spp., son un recurso biológico importante, para ser utilizado con eficacia en la agricultura sostenible, a través de la interacción planta-suelo.

La eficacia del microorganismo es lo más importante para la producción de bioinsumos y es la esencia de los productos biológicos. Este insumo puede representar hasta el $70 \%$ de la estructura de costos del producto final; en promedio, se estima que es del orden del 45 - 50\%, para los biofertilizantes y en los bioplaguicidas, está entre el 25 - 30\% (Rodríguez, 2003). Según Nakkeeran et al. (2006), normalmente, el aislamiento se realiza a partir de suspensiones de suelo, que tienen un consorcio de microorganismos, por lo tanto, la selección de una cepa eficaz es un criterio primordial, para el desarrollo de la agricultura.

Principales cultivos de aplicación: Los bioinsumos producidos e importados en el país están destinados, principalmente, a cultivos ornamentales, $24,7 \%$, seguido de bioinsumos para cultivos de hortalizas, $16,3 \%$ y de cereales, $13,2 \%$; el menor porcentaje de bioinsumos disponibles en el mercado fue para cultivos de tubérculos, 6,84\%; algodonero, 5,78\% y, café $3,68 \%$. El desarrollo y registro de bioinsumos para cultivos ornamentales está dado por el crecimiento, desde el año 2000, que tuvo la floricultura, alcanzando Colombia el segundo lugar, como exportador de flores frescas cortadas en el mundo, con una participación del $13 \%$, en el comercio total después de Holanda (Asocolflores, 2004).

Las condiciones sociales y ambientales en las cuales se producen las flores son cada vez más relevantes en los mercados internacionales. La preocupación por el impacto ambiental de la actividad productiva de flores de corte y las condiciones de los trabajadores, ha dado origen a una diversidad de iniciativas, que demandan la sustitución de los insumos de síntesis química (Asocolflores, 2004).

Análisis de los casos de patentes de invención de bioinsumos de uso agrícola inscritas en Colombia: Se identificaron 38 registros de patentes de invención, que contienen 
Cuadro 1. Microorganismos empleados para el desarrollo de bioinsumos registrados ante el ICA.

\begin{tabular}{|c|c|c|c|}
\hline MICROORGANISMO & No. de bioinsumos & MICROORGANISMO & No. de bioinsumos \\
\hline \multicolumn{2}{|c|}{ HONGOS MICORRÍZICOS } & \multicolumn{2}{|c|}{ HONGOS FILAMENTOSOS } \\
\hline Acaulospora sp. & 5 & Aspergillus niger & 1 \\
\hline A. laevins & 1 & A. oryzae & 1 \\
\hline A. longula & 1 & Beauveria sp. & 3 \\
\hline A. morrowiae & 1 & B. brongniartii & 1 \\
\hline A. foveata & 1 & B. bassiana & 21 \\
\hline Entrophospora sp. & 4 & Entomophthora virulenta & 1 \\
\hline E. schenckii & 1 & Gliocladium sp. & 1 \\
\hline E. colombiana & 1 & Lecanicillium sp. & 3 \\
\hline Gigaspora sp. & 3 & L. lecanii & 8 \\
\hline G. margarita & 1 & Metarhizium sp. & 5 \\
\hline Glomus sp. & 7 & Metarhizium anisopliae. & 16 \\
\hline G. fasciculatum & 1 & Nomuraea sp. & 1 \\
\hline G. occultum & 1 & N. rileyi & 2 \\
\hline G. clarum & 1 & Paecilomyces sp. & 3 \\
\hline Sclerocystis sp. & 1 & P. lilacinus & 12 \\
\hline Scutellospora sp. & 4 & P. fumosoroseus & 2 \\
\hline S. gilmore & 1 & Trichoderma sp. & 8 \\
\hline & & T. exiguum & 1 \\
\hline \multicolumn{2}{|c|}{ LEVADURAS } & T. harzianum & 18 \\
\hline Saccharomyces sp. & 2 & T. lignorum & 2 \\
\hline S. cerevisiae & 5 & T. pretiosum & 1 \\
\hline & & T. atroviride & 1 \\
\hline \multicolumn{2}{|c|}{ PARÁSITOS } & & \\
\hline Steinernema feltiae & 1 & \multicolumn{2}{|c|}{ BACILOS GRAM NEGATIVOS } \\
\hline & & Acetobacter diazotrophicus & 1 \\
\hline \multicolumn{2}{|c|}{ BACILOS GRAM POSITIVOS } & Azospirillum sp. & 1 \\
\hline Bacillus cereus & 1 & A. brasilense & 2 \\
\hline B. firmus & 2 & Azotobacter chroococcum & 3 \\
\hline B. mycoides & 1 & Bradyrhizobium sp. & 1 \\
\hline B. pumilus & 1 & B. japonicum & 4 \\
\hline B. subtilis & 3 & Burkholderia cepacia & 2 \\
\hline B. thuringiensis & 6 & Pseudonoma fluorescens & 2 \\
\hline B. thuringiensis var. kurstaki & 2 & Rhizobium sp. & 1 \\
\hline Lactobacillus acidophilus & 1 & Rhodopseudomonas palustres & 2 \\
\hline L. brevis & 1 & & \\
\hline L. casei & 3 & & \\
\hline
\end{tabular}


la palabra microorganismo. Luego de realizar la revisión de las patentes, seleccionando a las que corresponden a bioinsumos de uso agrícola, se identificaron 13 patentes, es decir, se presentan en promedio 1,75 solicitudes de patente por año.

La distribución de las patentes, de acuerdo al área de conocimiento registrada, se encuentra en el $64,3 \%$, como biotecnología, seguido por ingeniería química, con el 21,43\% y, con el 14,3\% química pura. Aunque el porcentaje de patentes registradas en el área de conocimiento biotecnología es alto, frente al universo de las patentes presentadas en Colombia, es muy pequeño. Según Dood \& Ruiz-Lozano (2012), el primer requisito para una iniciativa, a nivel empresarial en biotecnología, debería ser una solicitud de patente de la cepa identificada, así como el control de calidad, para mantener la confianza de los agricultores sobre la eficacia de la cepa; sin embargo, en Colombia antes de la modificación de la Decisión 486, no era posible solicitar patentes sobre los microorganismos.

Se revisó el IPC, que fue asignado a las patentes identificadas, para verificar que estuvieran asociadas a la producción de bioinsumos agrícolas. Entre los IPC registrados ante la SIC, se halló que seis pertenecen a bioquímica, cuatro de fertilizantes, dos de tratamiento del agua y uno de colorantes y resinas naturales. Este comportamiento porcentual y distribución grupal es similar a lo observado por Albornoz et al. (2007), en cuanto a la distribución de las patentes otorgadas en el área de biotecnología en Brasil, según la clasificación IPC a tres dígitos; el total acumulado, entre 2000 y 2007, presenta una fuerte concentración en el grupo C12, con 3.415 patentes, equivalentes al $63 \%$. Igualmente, Baquero et al. (2007) encontraron, a nivel mundial, las patentes distribuidas en dos clasificaciones, principalmente, A01N y $\mathrm{C} 12 \mathrm{~N}$, que corresponden a biopesticidas y microorganismos o enzimas, respectivamente.

Uno de los nodos establecidos representa la relación entre Qteros y la Universidad de Massachusetts. Esta alianza patentó la producción de etanol celulósico, a partir de residuos de maíz, de mijo y de bagazo de caña, utilizando el microorganismo $Q \circledR$ Clostridium phytofermentans. Esto coincide con lo reportado por Bisand et al. 2009, quienes identificaron contribuciones, tanto científicas como tecnológicas, provenientes de los países latinoamericanos, insertos en proyectos y redes, cuyos nodos residen en economías desarrolladas. El grueso de los temas de las agendas de investigación responde a las demandas de tales sociedades. En este sentido, los avances científicos, en este campo de la tecnología, tienen una clara y predeterminada utilización en actividades productivas concretas (Bisand et al. 2009).
Se pudo observar que sólo nueve patentes se encuentran dentro de las clasificaciones IPC reconocidas por la OCDE; sin embargo, cabe mencionar que el objetivo del enfoque de la OCDE es la biotecnología moderna, en oposición a los usos tradicionales de la utilización de organismos vivos, en los procesos de producción (Albornoz et al. 2007). Pese a ello, los únicos países latinoamericanos miembros de la OCDE son Chile y México.

Las cinco patentes que no se encuentran dentro de la clasificación OCDE corresponden a fertilizantes y composiciones no previstas en otro lugar. Luego de revisar el contenido de las patentes correspondían a biofertilizantes; esta clasificación inadecuada, se puede atribuir a que estas fueron las primeras presentadas entre 1997-2000 y no se tenía un conocimiento acerca de su vínculo con la biotecnología.

\section{Análisis de los trámites de acceso a recurso genético para} la investigación o desarrollo: La base de datos de acceso a recurso genético del MADS permitió identificar 103 solicitudes. Luego de realizar la selección de los que corresponden a microorganismos vinculados a la producción agrícola, se redujo a 16 registros.

No fue posible identificar si el origen de los microorganismos utilizados para el desarrollo de bioinsumos producidos en Colombia corresponde a aislamientos nativos o cepas introducidas, debido a que no se encontraron registros de solicitudes de acceso a recurso genético autorizando la investigación o su uso, con fines comerciales y una propuesta de distribución de beneficios económicos.

El microorganismo que presenta registro en las tres instituciones corresponde a Bacillus thuringiensis, sin embargo, al verificar cada uno de los registros ante el ICA, se observó que son cepas aisladas, por cada una de las empresas, que generaron el bioinsumo y no se encontraron relacionadas; el acceso a recurso genético fue concedido con fines de investigación científica, por lo tanto, no se logró generar un bioproducto, derivado de las mismas. Por último, la patente registrada es una mezcla para biodegradar materia orgánica compuesta por varios géneros de microorganismos, lo que indica que no fue registrada ante el ICA para su comercialización.

Ese es el caso de $B$. thuringiensis, catalogado como un pesticida tradicional. Los bio-plaguicidas basados en este microorganismo, se han utilizado en todo el mundo, pero la mayoría de las cepas son de tipo silvestre, que tienen un estrecho espectro de actividad insecticida (Jia et al. 2014). A menudo, se emplea la tecnología recombinante para mejorar la eficacia y reducir los costos de producción. 
Esta investigación muestra que en Colombia, el desarrollo de bioinsumos de uso agrícola, desde sus etapas iniciales hasta los procesos de desarrollo de patentes relacionados con los procesos de producción, ha pasado por alto los procesos para potenciar el uso de la biodiversidad microbiana.

Los procesos de generación de bioinsumos no están promoviendo el desarrollo de nuevos procesos de producción, medios de cultivo, presentaciones y formas de aplicación, que se constituyan en patentes para los investigadores. Según Bhattacharyya \& Jha (2012), la tendencia para el desarrollo de este tipo de productos está dirigida hacia la obtención de consorcios microbianos o microorganismos individuales, seleccionados con múltiples modos de acción, que permitan solucionar, al mismo tiempo, los problemas de fertilidad del suelo y el control de plagas y enfermedades.

En conclusión, se recomienda realizar entre las empresas productoras e importadoras de bioinsumos un análisis comparativo de los diversos parámetros, que definen la calidad mínima requerida de un producto, los cuales, deben ser establecidos para la supervisión del ICA y que la normatividad no ha contemplado hasta el momento. Se pudo determinar que algunos criterios que se pueden incluir, como requisito para registro de un bioinsumo, son el análisis genético de las cepas microbianas y el origen de las mismas, así como el depósito de los microorganismos ante una autoridad internacional acreditada, de acuerdo al Tratado de Budapest, facilitando la protección de la propiedad industrial.

Agradecimientos: Los autores agradecen a la Subgerencia de Protección Vegetal del ICA y a la Dirección Técnica de Inocuidad e Insumos Agrícolas, por su colaboración y disposición. Conflicto de intereses: El manuscrito fue preparado y revisado con la participación de todos los autores, quienes declaramos que no existe conflicto de intereses que ponga en riesgo la validez de los resultados presentados.

\section{BIBLIOGRAFÍA}

1. ALBORNOZ, M.; VISMARA, F.; LUCHILO, L. 2007. Catálogo de Patentes sobre Biotecnología en el MERCOSUR. BIOTECSUR. 59p.

2. ARÉVALO, E. 2009. Más arroz a menos costo con la aplicación de biofertilizantes y materia orgánica. Rev. Arroz. 57(476):26-28.

3. ASOCOLFLORES. 2004. Utilización de bioinsumos en Colombia: Estudio de Caso en flores de Corte. 60p.

4. BAQUERO, I.; TOBAR, M.; CAMPOS, S.; SUÁREZ, E.; ROSILLO, A.; SÁNCHEZ, J.; LANDÍNEZ, L. 2007.
Informe de vigilancia tecnológica. Bioinsumos. Programa Nacional de Biotecnología y Programa Nacional de Ciencias Agropecuarias. Departamento Administrativo de Ciencia, Tecnología e Innovación. Bogotá, Colombia. 145p.

5. BHATTACHARYYA, P.; JHA, D. 2012. Plant growth-promoting rhizobacteria (PGPR) emergence in agricultura. World. J. Microb. Biot. 28:1327-1350.

6. BISAND, R.; CAMPI, M.; CESA, V. 2009. Biotecnología y desarrollo. Comisión Económica para América Latina y el Caribe-CEPAL. Naciones Unidas. Santiago de Chile. 107p.

7. CABRERA, M.; LÓPEZ, C. 2008. Enfrentando los problemas de acceso: protegiendo las fuentes, mientras que se brinda certeza a los usuarios. UICN. Gland, Suiza. 81p.

8. CHENG, X.; LIU, C.; YAO, JW. 2010. The Current Status, Development Trend and Strategy of the Bio-pesticide Industry in China. Hubei. Agric. Sci. 49:222872290.

9. CONPES 3577 - CONSEJO NACIONAL DE POLÍTICA ECONÓMICA Y SOCIAL. 2009. Política nacional para la racionalización del componente de costos de producción asociado a los fertilizantes en el sector agropecuario. Bogotá, Colombia.33p. Disponible desde Internet en: http://www.ica.gov. co/getattachment/b527d0c9-e862-4c26-8347e5076fd9b1a9/2009CP3577.aspx (con acceso $18 / 08 / 2014)$.

10. CORTÉS, D.; VARGAS, J. 2012. Inequidad regional en Colombia. Serie de Documentos de trabajo No. 127. Facultad de Ciencias Económicas. Universidad del Rosario. Bogotá, Colombia. 82p.

11. DECRETO 309. PRESIDENCIA DE LA REPÚBLICA. 2000. Por el cual se reglamenta la investigación científica sobre diversidad biológica. Colombia. 10p.

12. DECRETO 302. PRESIDENCIA DE LA REPÚBLICA. 2003. Por el cual se modifica el parágrafo $1^{\circ}$ del artículo segundo del Decreto 309 de 2000, el cual reglamenta la investigación científica sobre diversidad biológica. Colombia. 2p.

13. DECRETO 1376. MINISTERIO DE AMBIENTE Y DESARROLLO SOSTENIBLE. 2013. Por el cual se reglamenta el permiso de recolección de especímenes 
silvestres de la diversidad biológica con fines de investigación científica no comercial. Colombia. 12p.

14. DÍAZ, M.; GUZMÁN, M.; OREA, U. 2007. Estudio patentométrico de un proyecto de investigación. Ciencias de la Información. Instituto de Información Científica y Tecnológica Cuba Red de Revistas Científicas de América Latina, El Caribe, España y Portugal. 38(12):57-66.

15. DOOD, I.; RUIZ-LOZANO, J. 2012. Microbial enhancement of crop resource use efficiency. Curr. Opin. Biotech. 23:236-242.

16. GARCÍA-OLIVARES, J.G.; MENDOZA-HERRERA, A.; MAYEK-PEREZ, N. 2012. Efecto de Azospirillum brasilense en el rendimiento del maíz en el norte de Tamaulipas, México. Universidad y Ciencia. 28(1):79-84.

17. GÓNGORA, C.; MARÍN, P.; BENAVIDES, M. 2009. Claves para el éxito del hongo Beauveria bassiana como controlador biológico de la broca del café. Avances Técnicos Cenicafé No. 384. Federación Nacional de Cafeteros de Colombia. Chinchiná, Caldas. Colombia. $8 p$.

18. HODSON DE JARAMILLO, E.; DÍAZ-ARIZA, L. 2013. Uso de bioinoculantes en la agricultura: Alternativa de manejo sostenible. En: Hodson de Jaramillo, E., Zamudio, T. Biotecnologías e Innovación: el compromiso social de la ciencia. Ed. Pontificia Universidad Javeriana. Bogotá, Colombia. 327p.

19. JIA, Y.; ZHAO, C.; WANG, Q.; SHU, C.; FENG, X.; SONG, F.; ZHANG, J. 2014. A genetically modified broad-spectrum strain of Bacillus thuringiensis toxic against Holotrichia parallela, Anomala corpulenta and Holotrichia oblita. World. J. Microbiol. Biotechnol. 30:595-603.

20. LENG, P.; ZHANG, Z.; PAN, G.; ZHAO, M. 2011. Applications and development trends in biopesticides. Afr. J. Biotechnol. 10(86):19864-19873.

21. MONTOYA, D. 2010. Avances en biotecnología: Panorama y perspectivas. En: Sánchez, G. Uribe, M. 2013. El desafío de generar tecnología en el siglo XXI. La propiedad intelectual en el devenir histórico de Colombia. Cátedra Manuel Ancízar. Universidad Nacional de Colombia, Bogotá. p.181-201.

22. NAKKEERAN, S.; FERNANDO, W.; SIDDIQUI, Z. 2006. Plant growth promoting rhizobacteria formulations and its scope in commercialization for the mana- gement of pests and diseases. In: Siddiqui, Z (Ed). 2011. PGPR: biocontrol and biofertilization. Springer, Dordrecht. p.257-296.

23. NEMOGÁ, G. 2009. Acceso a recursos Genéticos y su relación con Derechos de Propiedad Intelectual. En: Chaparro, A. 2009. Propiedad Intelectual en la era de los cultivos transgénicos. Universidad Nacional de Colombia, Bogotá. p.35-56.

24. NEMOGÁ, G. 2010. Biotecnología y el acceso a recursos genéticos. En: Sánchez, G., Uribe, M. (eds.). El desafío de generar tecnología en el siglo XXI. La propiedad intelectual en el devenir histórico de Colombia. Cátedra Manuel Ancízar. Universidad Nacional de Colombia, Bogotá. p.181-201.

25. NEMOGÁ, G.; ROJAS, D.; LIZARAZO, O. 2014. Investigación de la biodiversidad en países megadiversos: estrategias para alianzas científicas y técnicas. En: Ríos, M; y Mora, A, (eds), Acceso a recursos genéticos en América Latina y el Caribe: investigación, comercialización y cosmovisión indígena. UICNPNUMA/GEF-ABS-LAC. Quito, Ecuador. p.13-42.

26. KADIAN, N.; YADAV, K.; AGGARWAL, A. 2013. Significance of bioinoculants in promoting growth, nutrient uptake and yield of Cyamopsis tetragonoloba (L.) “Taub.”. Eur. J. Soil. Biol. 58:66-72.

27. PENNA, C.; MASSA, R.; OLIVIERI, F.; GUTKIND, G.; CASSÁN, F. 2011. A simple method to evaluate the number of bradyrhizobia on soybean seeds and its implication on inoculant quality control. AMB Express. 19(1):21.

28. RESOLUCIÓN ICA 698. 2011. Por medio de la cual se establecen los requisitos para el registro de departamentos técnicos de ensayos de eficacia, productores e importadores de bioinsumos de uso agrícola y se dictan otras disposiciones. 42p.

29. RESOLUCIÓN MINISTERIO DE AMBIENTE Y DESARROLLO SOSTENIBLE 068. 2002. Por la cual se establece el procedimiento para los permisos de estudio con fines de investigación científica en diversidad biológica y se adoptan otras determinaciones. Colombia. 8p.

30. RODRÍGUEZ, R. 2003. Estudios de mercados de la Biodiversidad. Informe final presentado al Instituto Alexander von Humboldt. Abril. Bogotá, Colombia. $114 p$. 
31. RUÍZ, M. 2010. Una lectura crítica de la Decisión 391 de la Comunidad Andina y su puesta en práctica en relación con el Tratado Internacional. Recursos Naturales y Ambiente. 53:136-147.

\section{SECRETARIA DEL CONVENIO DE DIVERSIDAD BIO-} LÓGICA. 2011. Protocolo de Nagoya sobre acceso a los recursos genéticos y participación justa y equitativa en los beneficios que se deriven de su utilización al Convenio sobre la diversidad biológica. Programa de Naciones Unidas para el Medio Ambiente. Montreal, Canadá. 16p.

33. SHIRI-JANAGARD, M.; RAEI, Y.; GASEMI-GOLEZANI, K.; ALIASGARZARD, N. 2012. Influence of Bradyrhizobium japonicum and phosphate solubilizing bacteria on soybean yield at different levels of nitrogen and phosphorus. Intl. J. Agron. Plant. Prod. 3(11):544549.

34. TORO, C. 2010. Tratados internacionales y biodiversidad. En: Sánchez, G., Uribe, M. 2013. El desafío de generar tecnología en el siglo XXI. La propiedad intelectual en el devenir histórico de Colombia. Cátedra Manuel Ancízar. Universidad Nacional de Colombia, Bogotá. p.181-201.

Recibido: Mayo 29 de 2014

Aceptado: Octubre 21 de 2014

\section{Cómo citar:}

Zambrano-Moreno, D.C.; Ramón-Rodríguez, L.F.; Van Strahlen-Pérez, M.; Bonilla-Buitrago, R.R. 2015. Industria de bioinsumos de uso agrícola en Colombia. Rev. U.D.C.A Act. \& Div. Cient. 18(1): 59-67. 